\title{
METHOD OF FORMULATING INPUT PARAMETERS OF NEURAL NETWORK FOR DIAGNOSING GAS-TURBINE ENGINES
}

\author{
Mykola Kulyk $^{1}$, Sergiy Dmitriev ${ }^{2}$, Oleksandr Yakushenko ${ }^{3}$, Oleksandr Popov ${ }^{4}$ \\ National Aviation University, 1 Kosmonavta Komarova Ave., 03680 Kiev, Ukraine \\ E-mail: ${ }^{4}$ eduicao@nau.edu.ua (corresponding author)
}

Received 29 October 2012; accepted 20 May 2013

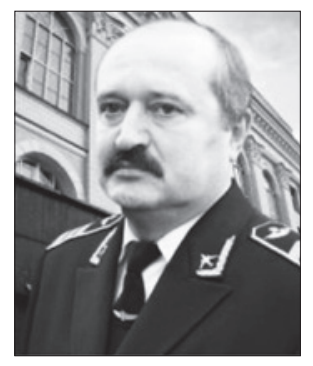

Mykola KULYK, Prof., Dr Sc., Eng.

Education: Kiev Institute of Civil Aviation Engineers, 1976. 1993 - Doctor of

Science (Engineering). 1994 - Professor.

Affiliations and functions: 1997 - head of the Department of Aircraft Engines,

2008 - rector of the National Aviation University.

Honours, awards: honoured person of Science and Engineering of Ukraine,

winner of the State Prize of Ukraine in the area of science and technology.

Present position: rector of the National Aviation University, Ukraine.

Research interests: automatic systems of aircraft engine technical conditions.

Publications: over 200 scientific papers.

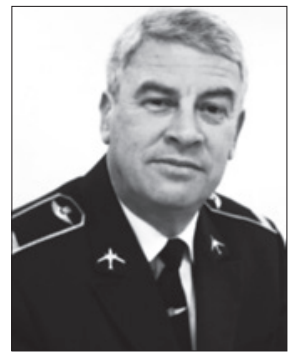

Sergiy DMITRIEV, Prof. Dr Sci. Eng.

Education: Kiev Institute of Civil Aviation Engineers, 1977. 1997 - PhD Engineering.

2000 - Professor.

Present position: head of the Department of Aircraft Continuing Airworthiness

Support, National Aviation University.

Research interests: diagnostics of the parameters of gas-turbine engines.

Publications: over 130 scientific papers.

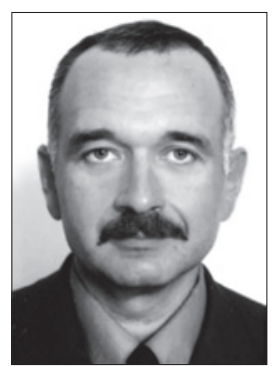

Oleksandr YAKUSHENKO, PhD Eng., Senior Researcher

Education: Kiev Institute of Civil Aviation Engineers, 1983. 2000 - PhD Engineering. Present position: associate professor in the Department of Aircraft Engines, National Aviation University.

Research interests: diagnostics, reliability of aviation engines.

Publications: 40 scientific papers.

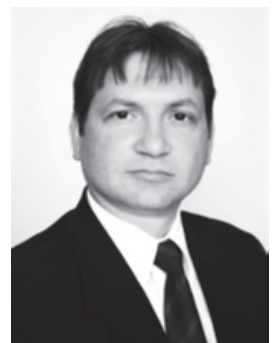

Oleksandr POPOV, PhD Eng., Assoc. Prof.

Education: National Aviation University, 2001. 2008 - PhD Engineering.

2011 - Associate Professor.

Present position: associate professor in the Department of Aircraft Continuing

Airworthiness Support, National Aviation University.

Research interests: diagnostics of the parameters of gas-turbine engines.

Publications: 40 scientific papers. 
Abstract. A method of obtaining test and training data sets has been developed. These sets are intended for training a static neural network to recognise individual and double defects in the air-gas path units of a gas-turbine engine. These data are obtained by using operational process parameters of the air-gas path of a bypass turbofan engine. The method allows sets that can project some changes in the technical conditions of a gas-turbine engine to be received, taking into account errors that occur in the measurement of the gas-dynamic parameters of the air-gas path. The operation of the engine in a wide range of modes should also be taken into account.

Keywords: gas-turbine engine, air-gas path, mathematical model of operational process, neural network.

\section{Introduction}

A way to improve the efficiency and reliability of diagnosis when assessing the technical conditions of the air-gas path of a gas-turbine engine is analysis of the parameters of operational process by using neural networks. The functioning of neural networks is however impossible without implementing the following pre-state processes: training, testing and network verification. The peculiarity of a neural network is its possibility of overfitting (Signal ... 1994; Advanced ... 1995). To solve this problem, the method of three sets of data can be used (Medvedev, Potiomkin 2002).

Each of these sets should characterise the full behaviour of the object that is being diagnosed in the classes of technical conditions being considered, such as a functioning engine, malfunctions of an engine, and combinations of the structural units in case of operational malfunctions of two interconnected units. The question is how to obtain these sets. In fact, it is necessary to have 20-200 or more calculated points to train the network to recognise a class of a technical condition. Each point includes diagnostic information for the air-gas path of a gas-turbine engine. This information is related to one of the possible combinations of the functional characteristics of the air-gas path units. The process of obtaining this information in a real operation can also be long. According to previous research, the only real source of the required information can be the results of a numerical experiment by using a mathematical model of the operational process of a gas-turbine engine. The data of real defects (malfunctions) can be used for obtaining a validation set. This paper considers a method of obtaining test and training sets for a static neural network.

\section{Determination of the technical conditions of a gas-turbine engine}

When obtaining the data sets, we used a nonlinear mathematical model of the operational process (second level of complexity). The formal description of the individual components of the air-gas path of a gas-turbine engine (compressor, combustor chamber, etc.) can also be used for the nonlinear mathematical model of the operational process. During the experiment, the vector of the mode parameters of the model $[R]$ is submitted to the input of a mathematical model of the operational process of an engine under a predetermined technical condition.
The output of the model is the desired parameters of the operational process obtained. The diagnostic parameters for training the network can be used as relative diagnostic deviations of these parameters:

$$
\bar{D}_{i}=\frac{P_{i}-P_{i}^{T}}{P_{i}^{T}},
$$

or

$$
\overline{D_{i}}=\frac{P_{i}-P_{i}^{T}}{\Delta P_{i}},
$$

where $\Delta P_{i}$ is the maximum measurement error of the first parameter; $P_{i}, P_{i}^{T}$ - values of the first parameter given for both a diagnosed gas-turbine engine and an average gas-turbine engine in the same mode and identical operating conditions:

$$
P=f^{\bmod }(R, a), P^{\mathbf{T}}=f^{\bmod }\left(R, a^{\mathbf{T}}\right),
$$

where $a, a^{\mathbf{T}}$ is the scale factors for the characteristics of the air-gas path units of a diagnosed gas-turbine engine and an average gas-turbine engine; $f^{\text {mod }}$ - a mathematical model of the engine.

Except for diagnostic deviations, a parameter characterising the operating conditions of a gas-turbine engine can be included in the set structure (revolutions per minute [rpm], for example). As an example of using this approach, the results received for the PS-90A aviation engine have been used (The engine ... 1990). The parameters of diagnostic deviations that are measured in flight have been included in the data sets for this engine: rpm of a high pressure rotor $n_{h p}$; total pressure behind the fan $\mathrm{P}_{\text {fan }}^{\star}$; total pressure behind the compressor $\mathrm{P}^{\star}{ }_{\mathcal{c}}$; temperature behind the compressor $\mathrm{T}^{*}{ }_{c}$; fuel consumption $\mathrm{G}_{\mathrm{f}}$; temperature behind the turbine $\mathrm{T}^{*}$; and the relation of total pressure behind the turbine to atmospheric pressure $\overline{P_{t}^{*}}$.

Besides those listed, the mode parameters of the model can be included in the set as well: rpm of the fan rotor $\mathrm{n}_{\mathrm{fan}}$; barometric height $\mathrm{H}$; Mach number of flight (M); and full temperature at the engine intake $\mathrm{T}^{*}$ in. The essential influence on the values of diagnostic deviations is one of the main conditions for each of parameters to be included in the set.

\section{Determination of the technical conditions of a gas-turbine engine during data set formation}

The method of scaling gas-turbine engine unit characteristics has been used to receive the parameters of an 
engine with a varied air-gas path. For example, to receive the individual characteristic of the turbine, we can use the following formula:

$$
\begin{aligned}
\mathrm{A}_{T} & =a_{A} \varphi_{T 1}\left(\left(\frac{\lambda_{U T}}{a_{U T}}\right),\left(\frac{\pi_{T}^{*}}{a_{\pi T}}\right)\right), \\
\eta_{T}^{*} & =a_{\eta} \varphi_{T 2}\left(\left(\frac{\lambda_{U T}}{a_{U T}}\right),\left(\frac{\pi_{T}^{*}}{a_{\pi T}}\right)\right),
\end{aligned}
$$

where $\varphi_{\mathrm{T} 1}, \varphi_{\mathrm{T} 2}$ is the characteristics of the turbine of an average gas-turbine engine; $\mathrm{A}_{\mathrm{T}}$ - parameter of the turbine flow; $\lambda_{U T}-$ the resulting circumferential speed of the turbine; $\eta_{T}^{\star} \pi_{T}^{*}$ - efficiency and pressure at gradient degree in the turbine; $a_{U T}, a_{\pi T}, a_{A}, a_{\eta}$ - scale factors under $\lambda_{U T}, \pi_{T}^{*}, A_{T}$ and $\eta_{T}^{*}$. For an average gas-turbine engine, each vector is individual. Two corrected scale coefficients have been selected for the description of the technical condition of each unit of the air-gas path. One of these scales is considered the main one, and the other is considered an auxiliary one. The main coefficient can be equally divided and vary within the limits $\Delta a_{i}^{\min }, \Delta a_{i}^{\max }$. This law has been selected to increase the quantity of the points around the line between defective and serviceable technical condition (TC) classes. Then the value of the first main coefficient is expressed as follows:

$$
\Delta a_{i}^{m}=\hat{f}^{u n i}\left(\Delta a_{i}^{\min }, \Delta a_{i}^{\max }\right),
$$

where $\hat{f}^{\text {uni }}$ is the generator of random numbers submitting to the uniform law with parameters $\Delta a_{i}^{\min }, \Delta a_{i}^{\max }$ for the first class.

Since variation in the values of the selected pair of factors has considerable correlation in the degradation of the air-gas path, one parameter is accepted as the main one, and the other as an auxiliary one. Both the auxiliary scale and the main one have statistical convergence.

The values of additional scales for the classes are expressed as:

$$
\Delta a_{i}^{a}=\hat{f}^{n o r m}\left(\Delta a_{i}^{m},\left|\Delta a_{i}^{m}\right| / K\right),
$$

where $\hat{f}^{\text {norm }}$ is the generator of random numbers that are under the normal law (the first parameter is a mathematical expectation, the second one is a standard deviation); $K$ - a constant (in calculations it is accepted as $K=6$ ). Since during malfunction turbine efficiency decreases while turbine flow increases, the value of the scale factor can be expressed as follows:

$$
\overline{\Delta a_{i}^{a}}=\hat{f}^{n o r m}\left(-\Delta a_{i}^{m},\left|\Delta a_{i}^{m}\right| / K\right) \text {. }
$$

A more detailed description of the model and characteristics of the scales is provided in reference (Ignatovich, Yakushenko 1997).

It is accepted that simultaneous occurrence of defects in two units of the air-gas path is possible for a gas-turbine engine. If one defect occurs, we can call the class of technical conditions primary, and if two defects occur, the class of technical conditions can be called secondary. The training set that describes the operation of an engine during take-off (rpm of a rotor $\mathrm{n}_{\mathrm{lp}}=4220 \mathrm{rpm}$ ) under standard atmospheric conditions is given in figure 1 .

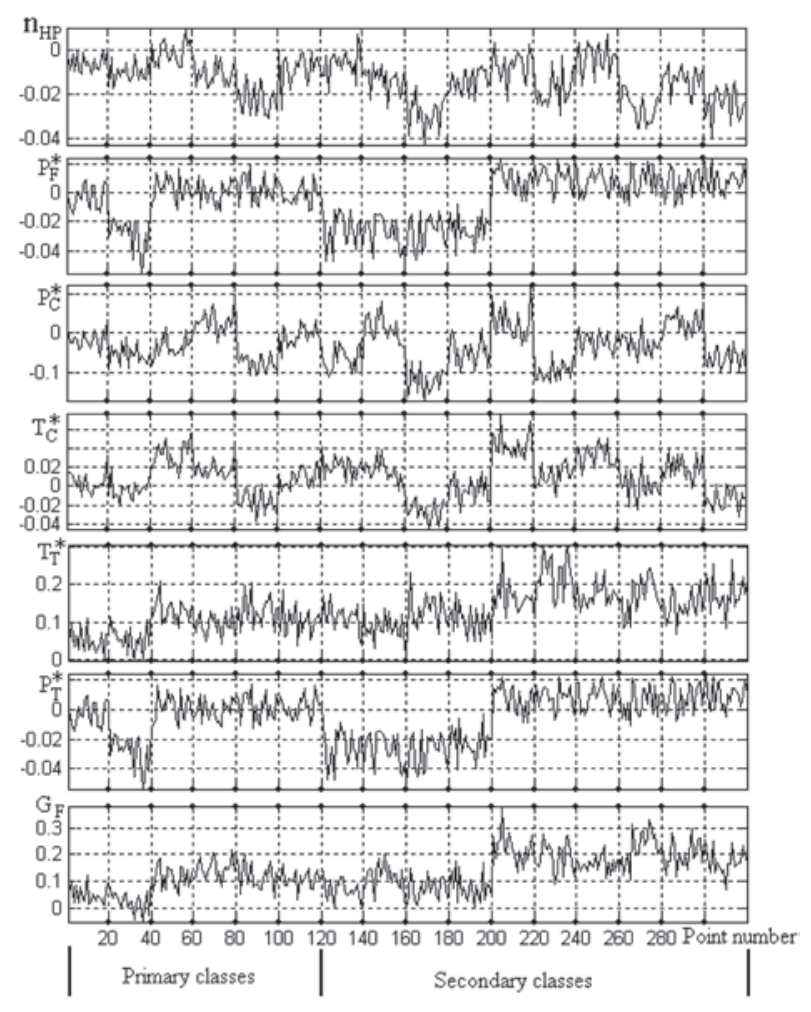

Fig. 1. The values of diagnostic deviation (Eq. (1)) in the training set for a neural network to recognize 16 of the TC classes

With the use of these data, qualitative analysis of the possibility of recognising primary classes is carried out. The direction and the displacement degree of diagnostic deviations of 2-6 classes (points 21-120) are analysed in accordance with the relative deviations of a class lacking defects (points 1-20). All primary classes are well enough spaced in the area of diagnostic deviations and can be distinguished.

The test set can be determined in the same way under the other initial conditions of the pseudo-random number generator.

\section{Determination of mode of operation of gas- turbine engine under external conditions}

Equation (1) or (2), as well as any other, can lead to a diagnostic deviation that depends on external conditions and the mode of operation of a gas-turbine engine.

All diagnostic modes should be equally presented to adequately distinguish the technical conditions of an engine. Then it is possible to receive the value of the first mode parameter of the model $\left(\mathrm{n}_{\mathrm{lp}}, \mathrm{H}, \mathrm{M}, \mathrm{T}^{*}{ }_{\text {in }}\right)$ according to the following expression:

$$
R_{j}=\hat{f}^{u n i}\left(R_{j}^{\min }, R_{j}^{\max }\right),
$$


where $R_{j}^{\min }, R_{j}^{\max }$ is the minimum and maximum value of the first mode parameter in diagnostic modes.

To accept that diagnostics are done during the takeoff phase, then the value $R_{j}^{\min }, R_{j}^{\max }$ is:

- for $n_{\mathrm{lp}}-100-2500 \mathrm{~m}$ above sea level (height of an airdrome location);

- for $\mathrm{M}-0-0.5$ (speed under initial rate of climb); - for $\mathrm{T}_{\text {in }}^{*}-238-313 \mathrm{~K}$.

\section{Determination of parameter measurement errors}

Errors and mistakes of measurement are the last factors that should be taken into account during the determination of sets for training of a neural network.

To receive the parameters of formula (1) and (2) (in our case $-\mathrm{n}_{\mathrm{hp}}, \mathrm{P}_{\text {fan }}^{\star}, \mathrm{P}_{\mathrm{c}}^{\star}, \mathrm{T}_{\mathrm{c}}^{*}, \mathrm{G}_{\mathrm{f}}, \mathrm{T}_{\mathrm{t}}^{\star}$ or $\mathrm{P}_{\mathrm{t}}^{\star}$ ), the following expression can be used:

$$
\begin{aligned}
& P_{i}=f^{\bmod }([R],[a])+\hat{f}^{n o r m}\left(0, \Delta P_{i} / 3\right), \\
& P_{i}^{T}=f^{\bmod }\left([\hat{R}],\left[a^{T}\right]\right),
\end{aligned}
$$

where $[\hat{R}]$ is a vector of measurement results of mode parameters (in our example $-\mathrm{n}_{\mathrm{hp}}, \mathrm{H}, \mathrm{M}, \mathrm{T}^{\star}{ }_{\text {in }}$ )

$$
\hat{R}_{i}=R_{i}+\hat{f}^{\text {norm }}\left(0, \Delta R_{i} / 3\right)
$$

The resulting training set is given in figure 2 . The set is supposed to consider a mode of operation of a gas-turbine engine and parameter measurement errors.

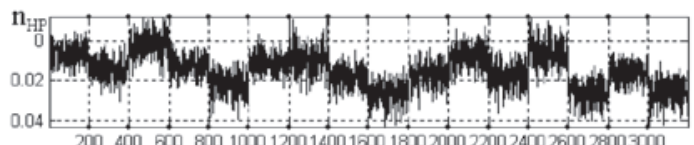

20040060080010001200140010001800200022002400260028003000
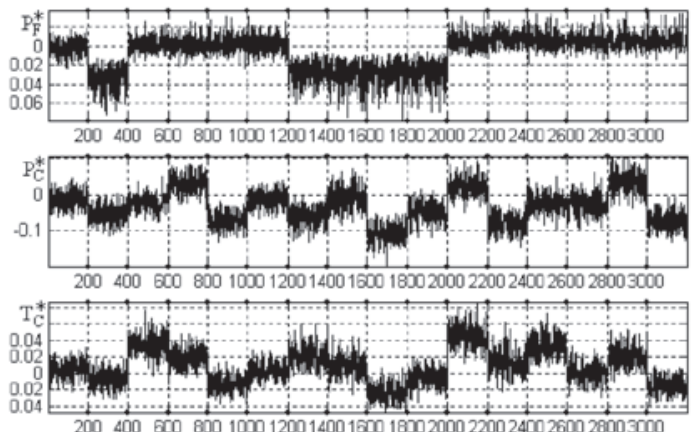

20040060000010001200140015001800200022002400260028003000

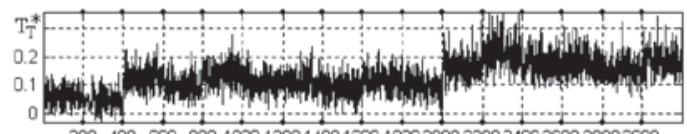

20040060080010001200140016001800200022002400260028003000

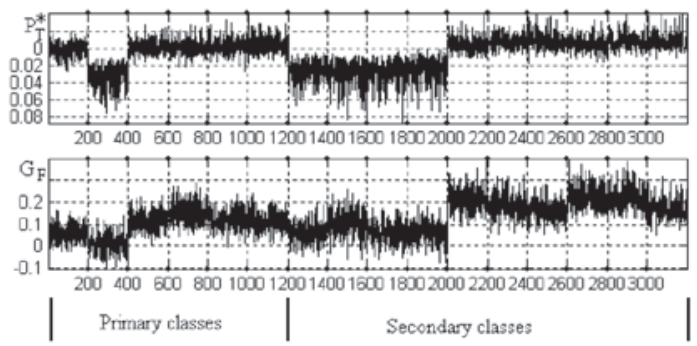

Fig. 2. The values of diagnostic deviations (Eq. (1)) in the test set for training a neural network to recognize 16 of the TC classes
Tables 1 and 2 show the correlation between mode parameters and diagnostic deviations calculated by Equation (1) and (2). All the coefficients of correlation are less than 0.15 . Thus, formula (1) gives smaller values of these coefficients. It specifies that mode parameters, in certain cases, may not be included in a set. This conclusion has been made by the results of mathematical modelling and should be compared with real flight data.

Table 1. Correlation factors between diagnostic deviations and mode parameters in use (1)

\begin{tabular}{|l|c|c|c|c|c|c|c|}
\hline & $N_{\mathrm{hp}}$ & $P_{\text {fan }}^{*}$ & $P_{\mathrm{c}}^{*}$ & $T_{\mathrm{c}}^{*}$ & $T_{t}^{*}$ & $\overline{P_{\mathrm{t}}^{*}}$ & $G_{f}$ \\
\hline$N_{\mathrm{lp}}$ & 0.0400 & 0.0038 & -0.0246 & -0.0364 & -0.0433 & -0.0362 & -0.0704 \\
\hline$T_{\mathrm{v}}^{*}$ & -0.0048 & -0.0077 & -0.0281 & -0.0152 & -0.0014 & 0.0036 & 0.0149 \\
\hline$H$ & 0.0006 & -0.0075 & 0.0261 & -0.0096 & -0.0258 & -0.0071 & -0.0059 \\
\hline$M$ & -0.0363 & -0.0583 & -0.0549 & -0.0504 & -0.0154 & -0.0576 & -0.0324 \\
\hline
\end{tabular}

Table 2. Correlation factors between diagnostic deviations and mode parameters in use (2)

\begin{tabular}{|l|c|c|c|c|c|c|c|}
\hline & $N_{\mathrm{hp}}$ & $P_{\text {fan }}^{*}$ & $P_{c}^{*}$ & $T_{\mathrm{c}}^{*}$ & $T_{t}^{*}$ & $\overline{P_{\mathrm{t}}^{*}}$ & $G_{f}$ \\
\hline$N_{\mathrm{lp}}$ & -0.0040 & -0.0192 & -0.1160 & -0.0191 & 0.0461 & -0.0563 & 0.2220 \\
\hline$T_{\mathrm{v}}^{*}$ & -0.0318 & -0.0023 & 0.0041 & -0.0031 & 0.0900 & 0.0083 & -0.0442 \\
\hline$H$ & 0.0018 & 0.0230 & 0.0902 & -0.0099 & -0.0276 & -0.0057 & -0.1421 \\
\hline$M$ & -0.0398 & -0.0677 & -0.0691 & -0.0479 & -0.0036 & -0.0477 & 0.0217 \\
\hline
\end{tabular}

If the objective is to train a neural network to reveal errors of measurement, it will be necessary to create a separate class in which all the classes of the technical condition are presented. Then each point of this class will have one or two parameters with an error of measurement more than $\Delta R_{i}$ or $\Delta P_{i}(i-$ parameter number):

$$
\begin{aligned}
& \hat{R}_{i}=R_{i} \pm \hat{f}^{\text {uni }}\left(\Delta R_{i}, K_{p} \cdot \Delta R_{i}\right), \\
\text { or } \quad & \hat{P}_{i}=f^{\bmod }([R],[a]) \pm \hat{f}^{u n i}\left(\Delta P_{i}, K_{p} \cdot \Delta P_{i}\right) .
\end{aligned}
$$

Thus, the diagnostic deviations and the mode parameters calculated according to Eqs (1), (3), (4), and (5) are included in the training and test sets.

\section{Conclusion}

A method of obtaining test and training data sets has been developed. It is important to stress that these data are intended for training a static neural network to recognise individual and multiple defects in the air-gas path units of a gas-turbine engine. Developing a method to optimise the structure of a neural network and select the best methods of training will be the following phases of investigation. 


\section{References}

Masters, T. 1995. Advanced Algorithms for Neural Networks. A C ++ Source-book. New York: Wiley. $431 \mathrm{p}$.

Ignatovich, S.; Yakushenko, A. 1997. The Projection of the Final Resource of a Gas-Turbine Engine by Means of Using a Mathematical Model of its Operational Process. Kharkov: Imis, 279-281.

Medvedev, V.; Potiomkin, V. 2002. Neural Networks. Matlab 6. Moscow: DIALOG-MIFI. $496 \mathrm{p}$.

Signal and Image Processing with Neural Networks by Timothy Masters: Masters T. Signal and Image Processing with Neural Networks. 1994. A C++ Sourcebook. New York: Wiley. 286 p.

The Engine PS-90A. 1990. Maintenance Manual. Perm: PNPO Aviadvigatel. 